service delivery and is already funding training, staff and services.

- District hospitals in Malawi are showing strong interest in developing palliative care services

- Project stakeholders are increasingly seeking extra funding and gifts in kind to support and develop children's palliative care services.

Discussion The collaborative implementation of this project has proved to be a catalyst for further development of palliative care than originally anticipated.

We would like to share our collaborative approach, together with more detailed results to inspire and encourage others to engage in partnerships to maximise impact and rise to the great challenge of meeting the huge need for palliative care internationally.

\section{P96 A SURVEY FOR CHILDREN/YOUNG PEOPLE SUPPORTED BY A HOSPICE}

Jo Stanford, Ruth Phillips. Shooting Star CHASE, Surrey, England

\subsection{6/bmjspcare-2013-000591.118}

A survey for children/young people supported by a hospice Introduction The Care Quality Commission requires agencies to enable services users to participate in the development of the service. Aims This survey represents a children's hospice first attempt to hear directly from the children and young people they support as part of the organisations Clinical Governance process.

Methods Children and young people using hospice services have a wide range of needs, abilities and disabilities. Participants were encouraged to complete the surveys on their own if able or if needed with help from their siblings or parents/carers. The format was a simple questionnaire provided on paper and accessible on-line to offer participants a choice about how to reply.

Completed paper surveys were added to the on-line programme manually by the Clinical Governance Facilitators to aid collation and analysis.

Results Of the 438 forms sent out 67 (15\%) were retuned; some completed by the child/young person, on their behalf by siblings or parent/carer or siblings from their own perspective.

Respondents commented on activities they would like to see offered by the hospice such as DJ evenings and outings including holidays abroad. They also highlighted a few things they didn't like such as staying over at the same time as young children.

Discussion The survey offered children and young people with a range of complex needs the opportunity to comment on their experiences. The results will be shared with staff so they can discuss and consider ways of implementing changes to their practice in an effort to meet specific needs and improve the experiences of the service users.

Conclusion The survey has provided a starting point. In future we plan to review the survey and include other methods such as participant observation to offer more children and young people the chance to participate.

\section{P97 DEVELOPMENT OF THE ADVANCED NURSE PRACTITIONER ROLE IN HOSPICE CARE: A MEDICAL MODEL}

Claire Hookey, Jane Whelan. Douglas Macmillan Hospice, Stoke- on-Trent, United Kingdom

10.1136/bmjspcare-2013-000591.119
Background/Context The role of the Advanced Nurse Practitioner (ANP) encompasses the provision of advanced levels of clinical practice, knowledge and skills. Whilst involving aspects of education and research, it is firmly grounded in direct clinical care. Responsibilities include assessing, diagnosing, planning and implementing programs of evidence based care and treatment. The drive to reduce junior doctors' hours in hospitals in the last decade led to further development in the ANP role, with increasing autonomy and application of expertise ( RCN 2012). More recently, the ANP role has translated into hospice care, although generally these roles have developed within hospice nursing teams.

Aim Following the resignation of a Speciality Doctor it was decided to develop an innovative ANP role within an already established, experienced medical team.

Approach used An ANP role was defined for our local context with reference to national guidance and recommendations from the Royal College of Nursing. The role sits within the medical team, and involves the assessment and management of hospice patients, working autonomously but with support from the medical team. The ANP also participates in the medical on call rota, supported by the Consultant on call. The successful candidate had many years of nursing experience both in hospital and community, working at an advanced level within nursing.

Outcomes The ANP role challenges traditional professional boundaries. However, the ANP has integrated well into the medical team, and both medical and nursing staff have identified benefits from the skills and experience that the role has brought. The post has also provided a cost effective means of fulfilling many roles normally undertaken by a doctor.

Application to hospice practice Other hospices may wish to explore the development of Advanced Nurse Practitioner roles within their own medical teams.

\section{P98 DEVELOPING AN ADVANCED NURSE PRACTITIONER ROLE IN HOSPICE CARE: A WORKFORCE TRANSFORMATION PROJECT}

${ }^{1}$ Gina Starnes, ${ }^{2}$ Sally-Ann Marciano, ${ }^{3}$ Clodagh Sowton, ${ }^{4}$ Andy Burt, ${ }^{5}$ Elaine McDonough, ${ }^{6}$ Colin Twomey, ${ }^{7}$ Michelle Larden, ${ }^{8}$ Karen Taylor. ${ }^{1}$ St Catherine's Hospice, Crawley, UK, ${ }^{2}$ Skills for Health, ${ }^{3}$ Phyllis Tuckwell Hospice Farnham, ${ }^{4}$ St Barnabas House WORTHING, ${ }^{5}$ St Michaels Hospice Hastings, ${ }^{6}$ St Wilfrids Hospice EastbourneE, ${ }^{7}$ St Michaels Hospice Basingstoke, ${ }^{8}$ Martlets Hospice Brighton, ${ }^{9}$ Oakhaven Hospice Lymington, ${ }^{10}$ Woking and Sam Beare Hospice

\subsection{6/bmjspcare-2013-000591.120}

Twelve hospices from the ECLIHP Regional group working with Skills for Health as part of a successful bid for $£ 10,000$ foa a supported project.

The overall aim of this project will be to improve the quality of care for people with complex palliative and end of life care needs and their families by ensuring 24/7 direct admissions access to specialist inpatient hospice beds and emergency out of hours assessment in the community.

The intention of this project is to support delivery of a flexible and responsive 24/7 hospice admission and assessment service through redesign of the existing workforce to introduce an Advanced Nurse Practitioner (ANP) role within the team.

Impact will be measured by agreed Key Performance Indicators

Desired Outcome 